\title{
Asteroseismological constraints on the pulsating planetary nebula nucleus (PG 1159-type) RX J2117.1+3412
}

\author{
A. H. Córsico ${ }^{1,2, \star}$, L. G. Althaus ${ }^{1,2,} \star$, M. M. Miller Bertolami ${ }^{1,2,3, \star \star}$, and K. Werner ${ }^{4}$ \\ 1 Facultad de Ciencias Astronómicas y Geofísicas, Universidad Nacional de La Plata, Paseo del Bosque S/N, \\ (1900) La Plata, Argentina \\ e-mail: [acorsico;althaus;mmiller]@fcaglp.unlp.edu.ar \\ 2 Instituto de Astrofísica La Plata, IALP, CONICET-UNLP \\ 3 Max-Planck-Institut für Astrophysik, Garching, Germany \\ ${ }^{4}$ Institut für Astronomie und Astrophysik, Universität Tübingen, Sand 1, 72076 Tübingen, Germany \\ e-mail: werner@astro.uni-tuebingen.de
}

Received 26 September 2006 / Accepted 12 October 2006

ABSTRACT

\begin{abstract}
Aims. We present asteroseismological inferences on RXJ2117.1+3412, the hottest known pulsating PG 1159 star. Our results are based on full PG 1159 evolutionary models recently presented by Miller Bertolami \& Althaus (2006).

Methods. We performed extensive computations of adiabatic $g$-mode pulsation periods on PG 1159 evolutionary models with stellar masses ranging from 0.530 to $0.741 M_{\odot}$. PG 1159 stellar models are extracted from the complete evolution of progenitor stars started from the ZAMS, through the thermally pulsing AGB and born-again phases to the domain of the PG 1159 stars. We constrained the stellar mass of RX J2117.1+3412 by comparing the observed period spacing with the asymptotic period spacing and with the average of the computed period spacings. We also employed the individual observed periods to find a representative seismological model for RX J2117.1+3412.

Results. We derive a stellar mass $M_{*} \sim 0.56-0.57 M_{\odot}$ from the period spacing data alone. In addition, we found a best-fit model representative for RX $2117.1+3412$ with an effective temperature $T_{\text {eff }}=163400 \mathrm{~K}$, a stellar mass $M_{*}=0.565 M_{\odot}$, and a surface gravity $\log g=6.61$. The derived stellar luminosity and radius are $\log \left(L_{*} / L_{\odot}\right)=3.36$ and $\log \left(R_{*} / R_{\odot}\right)=-1.23$, respectively, and the He-rich envelope thickness is $M_{\text {env }}=0.02 M_{\odot}$. We derive a seismic distance $d \sim 452 \mathrm{pc}$ and a linear size of the planetary nebula $D_{\mathrm{PN}} \sim 1.72 \mathrm{pc}$. These inferences seem to solve the discrepancy between the RX J2117.1+3412 evolutionary timescale and the size of the nebula. All of the seismological tools we use concur to the conclusion that RX J2117.1+3412 must have a stellar mass $M_{*} \sim 0.565 M_{\odot}$, much in agreement with recent asteroseismology studies and in clear conflict with the predictions of spectroscopy plus evolutionary tracks.
\end{abstract}

Key words. stars: evolution - stars: interiors - stars: oscillations - planetary nebulae: general

\section{Introduction}

Pulsating PG 1159 stars (or GW Vir stars) are very hot hydrogen-deficient post-Asymptotic Giant Branch (AGB) stars with surface layers rich in helium, carbon and oxygen (Werner \& Herwig 2006). They exhibit multiperiodic luminosity variations with periods in the range 300-3000 s, attributable to nonradial pulsation $g$-modes. Some GW Vir stars are still embedded in a planetary nebula (commonly called PNNVs; Planetary Nebula Nucleus Variable), and are characterized by much higher luminosity than the "naked" GW Vir stars (those without nebulae). PG 1159 stars are thought to be the evolutionary link between post-AGB stars and most of the hydrogen-deficient white dwarfs. These stars are believed to be the result of a born again episode triggered either by a very late helium thermal pulse (VLTP) occurring in a hot white dwarf shortly after hydrogen burning has almost ceased (see Schönberner 1979 and more recently Herwig 2001; Lawlor \& MacDonald 2003; Althaus et al. 2005; and Miller Bertolami et al. 2006) or a late helium

* Member of the Carrera del Investigador Científico y Tecnológico, CONICET, Argentina.

$\star \star$ Fellow of CONICET, Argentina. thermal pulse (LTP) that takes place during the post-AGB evolution when hydrogen burning is still active (see Blöcker 2001, for references). During a VLTP episode, most of the hydrogen-rich envelope of the star is burnt in the helium flash-driven convection zone, while in a LTP the hydrogen deficiency is the result of a dilution episode. In both cases, the star returns rapidly back to the AGB and finally into the domain of high effective temperature as a hydrogen-deficient, quiescent helium-burning object. This helium burning phase continues until the hot white-dwarf domain is reached.

RXJ2117.1+3412 is the hottest known pulsating PG 1159 star. It was discovered as an X-ray source in the ROSAT sky survey (Motch et al. 1993). The star is embedded in an old, extended and diffuse planetary nebula, which at the distance of $1400 \mathrm{pc}$ (Appleton et al. 1993) makes it the largest presently known with a diameter of 5.3 pc. This implies an expansion age much in excess as compared with that predicted by stellar evolution (Appleton et al. 1993). For the central star Werner et al. (1996) and Rauch \& Werner (1997) derived, with the help of optical, UV, and X-ray spectra and NLTE model atmospheres, $T_{\text {eff }}=170 \pm 10 \mathrm{kK}$ and $\log g=6_{-0.2}^{+0.3}$, and a surface composition of $\mathrm{He} / \mathrm{C} / \mathrm{O}=47.5 / 23.8 / 6.2$ (by number of atoms). Furthermore, a strong mass-loss rate of $\dot{M} \approx 10^{-7}-10^{-8} M_{\odot} / \mathrm{yr}$ 
has been measured (Koesterke et al. 1998; Koesterke \& Werner 1998). The central star of RX J2117.1+3412 was discovered to be a pulsator independently by Watson (1992) and Vauclair et al. (1993), with pulsation periods that are intermediate between those of PNNVs and naked GW Vir stars. The effective temperature of the central star defines the blue edge of the observational PG 1159 instability strip. Vauclair et al. (2002) (hereinafter VEA02) published an amazing richness of asteroseismological results on RX J2117.1+3412 from a multisite photometric campaign that included two Whole Earth Telescope (WET, Nather et al. 1990) runs. Without actually performing pulsation computations, they were still able to derive fundamental quantities for RX J2117.1+3412, such as the stellar mass, the helium-rich envelope mass fraction, rotation rate, luminosity, and distance.

It is important to note that at the time the analysis of VEA02 was performed, no realistic PG 1159 evolutionary models suitable for interpreting high-luminosity, high-temperature transition objects such as RXJ2117.1+3412 were available. In fact, while the $0.7 M_{\odot}$ evolutionary sequence of Wood \& Faulkner (1986) available at that time fits the location of $\mathrm{RX} \mathrm{J} 2117.1+3412$ in the $\log g-\log T_{\text {eff }}$ plane, its predicted surface abundance composition is not representative of the observed abundances in RX J2117.1+3412. More importantly, the Wood \& Faulkner (1986) models do not constitute appropriate structures for PG 1159 models that have been evolved through a bornagain episode. On the other hand, the evolutionary tracks of Gautschy (1997), with surface abundances more appropriate to PG 1159 stars, do not have luminosities high enough to be compatible with RX J2117.1+3412. In view of the lack of realistic stellar models for a full asteroseismological analysis, VEA02 were forced to use the scaling relation between period spacing and mass of Kawaler \& Bradley (1994) to infer the stellar mass of RX J2117.1+3412. It is worth noting that this relation was derived for GW Vir stars characterized by much lower luminosities than those of the PNNVs.

One of the results of VEA02 is that the asteroseismological mass they derive for RX J2117.1+3412 is about $0.56 M_{\odot}$, in serious disagreement with the mass derived from spectroscopical analysis $\left(0.72 M_{\odot}\right)$. This fact has motivated us to undertake the present investigation taking full advantage of the new generation of PG 1159 evolutionary models recently developed by Miller Bertolami \& Althaus (2006). In fact, these authors have computed realistic PG 1159 evolutionary sequences for different stellar masses by considering the complete evolution of progenitor stars. They have followed in detail all of the evolutionary phases prior to the formation of PG 1159 stars, particularly the born-again stage. We believe that the evolutionary models presented by Miller Bertolami \& Althaus (2006) represent a solid theoretical background to analyze the evolutionary and pulsational status of hot PG 1159 stars like RX J2117.1+3412, a transition object for which stellar models calculated with artificial evolutionary procedures should be taken with caution.

In this work we perform an asteroseismological analysis of RX J2117.1+3412 based on the PG 1159 evolutionary models of Miller Bertolami \& Althaus (2006). Emphasis is placed on deriving pertinent structural parameters for this star by using its observed period spectrum. In particular, we derive the stellar mass for this object from three different seismological approaches. Additionally, the implications of our results for the evolutionary status of RX J2117.1+3412 and for its planetary nebula are discussed. The paper is organized as follows: in the next section we briefly describe the input physics of the PG 1159 evolutionary models and the pulsational code we employ. In Sect. 3 we derive the stellar mass of RX J2117.1+3412 by means of the observed period spacing. In Sect. 4 we derive structural parameters of this star by employing the individual observed periods. In Sect. 5 we discuss the discrepancy we found between the seismological and the spectroscopic mass of RX J2117.1+3412, and in Sect. 6 we discuss the thickness of the outer envelope of the star as predicted by our seismological model. The asteroseismological distance to RX J2117.1+3412 and the implications of our analysis for its planetary nebula are provided in Sect. 7. Finally, in Sect. 8 we summarize our main results and make some concluding remarks.

\section{Evolutionary models and numerical tools}

The pulsation analysis presented in this work relies on stellar models that take into account the complete evolution of PG 1159 progenitor stars. The pulsation stability properties of these PG 1159 models have recently been analyzed by Córsico et al. (2006). The stages for the formation and evolution of PG 1159 stars were computed with the LPCODE evolutionary code, which is described in detail in Althaus et al. (2005). Briefly, LPCODE uses OPAL radiative opacities (including carbon- and oxygen-rich mixtures) from the compilation of Iglesias \& Rogers (1996), complemented at the low-temperature regime with the molecular opacities of Alexander \& Ferguson (1994) (with solar metallicity). Chemical changes are performed via a time-dependent scheme that simultaneously treats nuclear evolution and mixing processes due to convection, salt finger and overshooting. Convective overshooting is treated as an exponentially decaying diffusive process above and below any convective region.

Specifically, the background of stellar models were extracted from the PG 1159 evolutionary calculations recently presented in Miller Bertolami \& Althaus (2006) $(0.53,0.542,0.565,0.609$ and $0.664 M_{\odot}$ sequences), Althaus et al. (2005) $\left(0.589 M_{\odot}\right)$ and Córsico et al. (2006) $\left(0.741 M_{\odot}\right)$. The range of initial masses of these models on the ZAMS is $1-3.75 M_{\odot}$. We refer the reader to those works for details. All of the sequences were followed from the ZAMS (assuming solar metallicities) through the thermally pulsing and mass-loss phases on the AGB. After experiencing several thermal pulses, the progenitors departed from the AGB and evolved towards high effective temperatures. The mass-loss rate during the AGB was arbitrarily kept fixed, as to obtain a final helium shell flash during the early white dwarf cooling phase (VLTP). As a result, evolution proceeds through the born-again stage, which brings the remnants back to the giant domain. After this episode, most of the hydrogen content of the stars was burnt, and the hydrogen-deficient remnants evolved at constant luminosity to the domain of PG 1159 stars with surface chemical composition rich in helium, carbon and oxygen. The evolutionary tracks in the $\log T_{\text {eff }}-\log g$ plane are displayed in Fig. 1. For the $1-M_{\odot}$ sequence two different AGB evolutions were considered, with different mass-loss rates as to obtain different numbers of thermal pulses and, eventually, two different remnant masses of 0.530 and $0.542 M_{\odot}$. Mass-loss episodes after the VLTP were not considered in the PG 1159 evolutionary sequences we employed here, despite the fact that mass-loss episodes for PG 1159 stars have been reported (see Werner \& Herwig 2006).

We computed adiabatic pulsation periods with the help of the numerical code we employed in our previous works (see Córsico \& Althaus 2006, for details). We analyzed about 3000 PG 1159 models covering a wide range of effective temperatures $\left(5.5 \gtrsim \log \left(T_{\text {eff }}\right) \gtrsim 4.8\right)$ and luminosities 


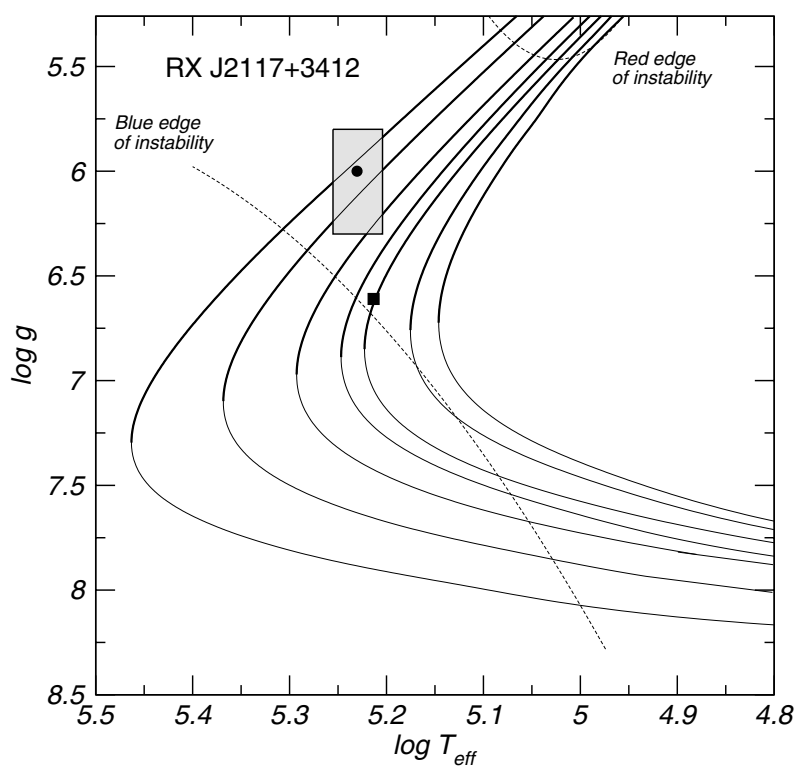

Fig. 1. The Miller Bertolami \& Althaus (2006) PG 1159 full evolutionary tracks in the $\log T_{\text {eff }}-\log g$ plane, corresponding to stellar masses of (from right to left): 0.530, 0.542, 0.565, 0.589, 0.609, 0.664, and $0.741 M_{\odot}$. Thick (thin) curves correspond to stages before (after) the models reach their highest effective temperature. The black circle with the error box is the location of RXJ2117.1+3412 according to spectroscopy $\left(T_{\text {eff }}=170 \pm 10 \mathrm{kK}\right.$ and $\left.\log g=6_{-0.2}^{+0.3}\right)$ and the black square is the location of the star as predicted by our asteroseismological analysis (Sect. 4). The blue (hot) and red (cool) boundaries of the theoretical dipole $(\ell=1)$ instability domain according to Córsico et al. (2006) are depicted with thin dashed lines.

$\left(0 \lesssim \log \left(L_{*} / L_{\odot}\right) \lessgtr 4.2\right)$, and a range of stellar masses $(0.530 \leq$ $\left.M_{*} / M_{\odot} \leq 0.741\right)$. For each model we computed $\ell=1 \mathrm{~g}$ mode periods ${ }^{1}$ in the range $50 \lesssim \Pi_{k} \lesssim 3600 \mathrm{~s}$, which comfortably covers the period spectrum observed in RX J2117.1+3412 (690-1200 s).

\section{Mass determination from the observed period spacing}

Here, we constrain the stellar mass of RX J2117.1+3412 by comparing the asymptotic period spacing $\left(\Delta \Pi_{\ell}^{\mathrm{a}}\right)$ and the average of the computed period spacings $\left(\overline{\Delta \Pi_{k}}\right)$ with the observed period spacing $\left(\Delta \Pi^{\mathrm{O}}\right)^{2}$. These methods take full advantage of the fact that the period spacing of PG 1159 pulsators depends primarily on the stellar mass (Kawaler \& Bradley 1994; Córsico \& Althaus 2006).

The asymptotic period spacing and the average of the computed period spacings for $\ell=1$ modes as a function of the effective temperature are displayed in Figs. 2 and 3, respectively, for different stellar masses. Also shown in these diagrams is the location of RXJ2117.1+3412, with $T_{\text {eff }}=170 \pm 10 \mathrm{kK}$ (Werner et al. 1996) and $\Delta \Pi^{\mathrm{O}}=21.618 \pm 0.008 \mathrm{~s}$ (VEA02). Here, $\Delta \Pi_{\ell}^{\mathrm{a}}=\Pi_{0} / \sqrt{\ell(\ell+1)}$, where $\Pi_{0}=2 \pi^{2}\left[\int_{r_{1}}^{r_{2}}(N / r) \mathrm{d} r\right]^{-1}$, being $N$ the Brunt-Väisälä frequency (Tassoul et al. 1990). The quantity $\overline{\Delta \Pi_{k}}$, on the other hand, is assessed by averaging the computed forward period spacings $\left(\Delta \Pi_{k}=\Pi_{k+1}-\Pi_{k}\right)$ in the range of the observed periods in $\mathrm{RX} \mathrm{J} 2117.1+3412$.

${ }^{1}$ Quadrupole $(\ell=2)$ modes have not been detected in RX J2117.1+3412 (VEA02).

${ }^{2}$ Note that the vast majority of asteroseismic studies are based on the asymptotic period spacing to infer the stellar mass of GW Vir pulsators.

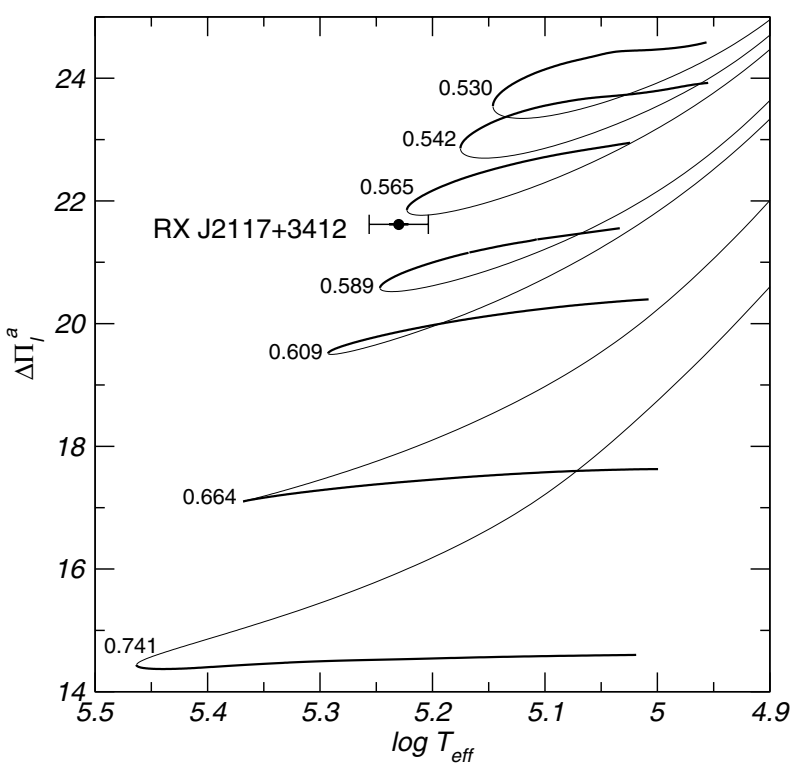

Fig. 2. The dipole $(\ell=1)$ asymptotic period spacing $\left(\Delta \Pi_{\ell}^{\mathrm{a}}\right)$ for different stellar masses in terms of the effective temperature. Numbers at the left of each curve denote the stellar masses (in solar units). Thick (thin) curves correspond to stages before (after) the models reach their highest effective temperature. The small black circle with the error bar depicts the location of RXJ2117.1+3412 $\left(T_{\text {eff }}=170 \pm 10 \mathrm{kK}\right.$ and $\Delta \Pi^{\mathrm{O}}=$ $21.618 \pm 0.008 \mathrm{~s})$.

From a comparison between $\Delta \Pi^{\mathrm{O}}$ and $\Delta \Pi_{\ell}^{\mathrm{a}}$ ("first method"), we obtain a stellar mass of $M_{*}=0.568_{-0.003}^{+0.008} M_{\odot}$. The quoted uncertainties in the value of $M_{*}$ come from the errors in the spectroscopic determination of the effective temperature ${ }^{3}$. Note that, since the $M_{*}=0.565 M_{\odot}$ evolutionary track does not reach the spectroscopic $T_{\text {eff }}$ of RXJ2117.1+3412 (see Figs. 1 and 2), we are forced to extrapolate the value of $\Delta \Pi_{\ell}^{\mathrm{a}}$ for that effective temperature $(170 \mathrm{kK})$ and also for its upper limit $(180 \mathrm{kK})$ to derive the stellar mass.

In the same way, we get $M_{*}=0.560_{-0.013}^{+0.018} M_{\odot}$ if we compare $\Delta \Pi^{\mathrm{O}}$ and $\overline{\Delta \Pi_{k}}$ ("second method"). Again, we need to extrapolate the values of $\overline{\Delta \Pi_{k}}$ for the spectroscopic value of $T_{\text {eff }}$ and its upper limit in order to derive the stellar mass and its lower limit.

It is evident that both estimations of the stellar mass nearly agree $^{4}$. The slightly higher value of $M_{*}$ as derived from $\Delta \Pi_{\ell}^{\mathrm{a}}$ is due to that usually the asymptotic period spacing is a bit larger than the average of the computed period spacings (see Córsico $\&$ Althaus 2006). The first method to derive the stellar mass is somewhat less realistic than the second one, because the asymptotic predictions are, in principle, only valid for chemically homogeneous stellar models, while our PG 1159 models are indeed chemically stratified (see Sect. 6).

${ }^{3}$ Our uncertainties are different from those quoted in VEA02 which are due entirely to the fact that they lack evolutionary calculations appropriate for RX J2117.1+3412.

${ }^{4}$ Note that in both derivations of $M_{*}$ we have assumed that RX J2117.1+3412 is evolving towards higher effective temperatures, before it reaches the evolutionary knee in the $\log T_{\text {eff }}-\log g$ diagram. In other words, we have used the thick portions of the curves in Figs. 2 and 3 to infer the stellar mass. Assuming instead that the star is on the beginning of its white dwarf cooling phase, then the thin portions of the curves should have been considered. In that case we would have obtained values of the stellar mass only slightly different from those quoted in the text. 


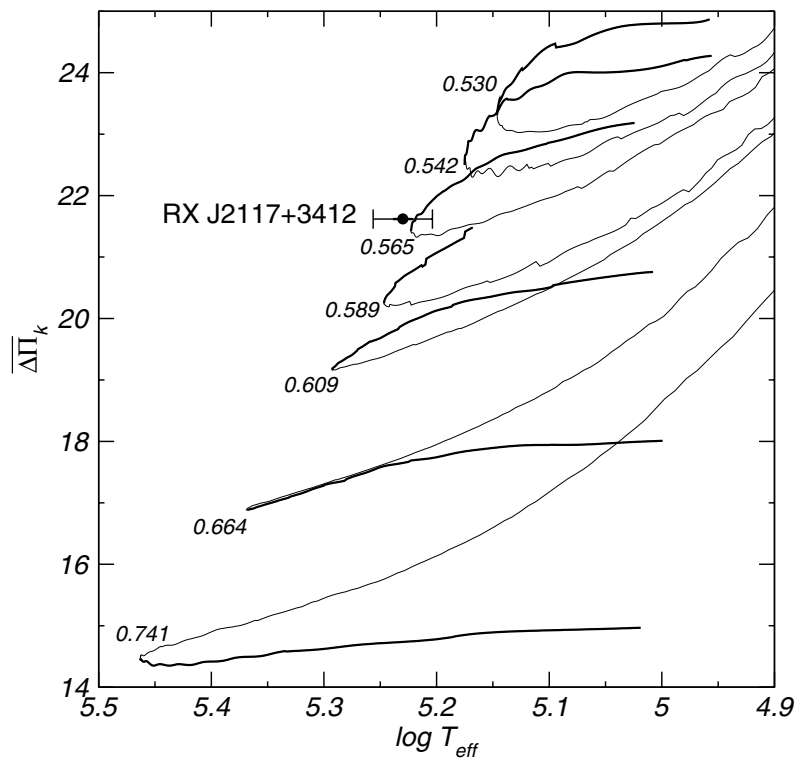

Fig. 3. Same as Fig. 2, but for the average of the computed period spacings $\left(\overline{\Delta \Pi_{k}}\right)$.

VEA02 derive a stellar mass of $M_{*}=0.56_{-0.04}^{+0.02} M_{\odot}$ by extrapolating $\Pi_{0}$ from the formula of Kawaler \& Bradley (1994). It is interesting to note that, despite that extrapolation being based on a relation which is valid for luminosities between $1.6 \leq \log \left(L_{*} / L_{\odot}\right) \leq 2.8$ (much lower than the luminosity of RX J2117.1+3412), the mass derived by VEA02 is in complete agreement with our predictions, although our uncertainties are much smaller.

Finally, we note that our inferred stellar mass value of $M_{*} \approx 0.56-0.57 M_{\odot}$, is in serious conflict with the value $M_{*}=0.72_{-0.12}^{+0.15} M_{\odot}$ as derived from spectroscopy coupled to evolutionary tracks ${ }^{5}$ (Miller Bertolami \& Althaus 2006). This will be discussed in more detail below.

\section{Constraints from the individual observed periods}

In this approach we seek a pulsation model that best matches the individual pulsation periods of RX J2117.1+3412. The goodness of the match between the theoretical $\ell=1$ pulsation periods $\left(\Pi_{k}\right)$ and the observed periods $\left(\Pi_{i}^{\mathrm{O}}\right)$ is measured by means of a quality function defined as $\chi^{2}\left(M_{*}, T_{\text {eff }}\right)=\sum_{i=1}^{n} \min \left[\left(\Pi_{i}^{\mathrm{O}}-\Pi_{k}\right)^{2}\right] / n$, where $n(=20)$ is the number of observed periods (first column in Table 1). The PG 1159 model that shows the lowest value of $\chi^{2}$ will be adopted as the "best-fit" model.

We evaluate the function $\chi^{2}\left(M_{*}, T_{\text {eff }}\right)$ for stellar masses $0.530,0.542,0.565,0.589,0.609,0.664$, and $0.741 M_{\odot}$, whereas for the effective temperature we employed a much more finer grid $\left(\Delta T_{\text {eff }}=10-30 \mathrm{~K}\right)$. The function $\chi^{2}$ in terms of the effective temperature for different stellar masses is shown in the mosaic of Fig. 4. We found only two important minima of comparable magnitude, one of them for the model with $M_{*}=0.565 M_{\odot}$ and $T_{\mathrm{eff}} \approx 163.4 \mathrm{kK}$ (panel c) and the other one for the model with $M_{*}=0.589 M_{\odot}$ and $T_{\text {eff }} \approx 105 \mathrm{kK}$ (panel d). Another minimum, albeit less pronounced, is also encountered for the model with $M_{*}=0.565 M_{\odot}$ at $T_{\text {eff }} \approx 123 \mathrm{kK}$. We adopt the first model as the best-fit model because it has an effective temperature relatively

\footnotetext{
5 The quoted uncertainties in the spectroscopic value of $M_{*}$ have their origin in the uncertainties in the determination of $\log g$ and $T_{\text {eff }}$, e.g., we neglect possible uncertainties in the evolutionary computations.
}

Table 1. Observed $m=0$ periods $\left(\Pi_{i}^{\mathrm{O}}\right)$ of RX $2117.1+3412$ (taken from VEA02), theoretical $\ell=1$ periods $\left(\Pi_{k}\right)$, period differences $\left(\delta \Pi_{i}\right)$, radial orders $(k)$, normalized growth rates $\left(\eta_{k}\right)$, and rates of period change $\left(\dot{\Pi}_{k}\right)$ of the best-fit model.

\begin{tabular}{cccccc}
\hline \hline $\begin{array}{c}\Pi_{i}^{\mathrm{O}} \\
{[\mathrm{s}]}\end{array}$ & $\begin{array}{c}\Pi_{k} \\
{[\mathrm{~s}]}\end{array}$ & $\begin{array}{c}\delta \Pi_{i} \\
{[\mathrm{~s}]}\end{array}$ & $k$ & $\begin{array}{c}\eta_{k} \\
{\left[10^{-7}\right]}\end{array}$ & $\begin{array}{c}\dot{\Pi}_{k} \\
{\left[10^{-11} \mathrm{~s} / \mathrm{s}\right]}\end{array}$ \\
\hline 692.27 & 689.77 & 2.5 & 30 & -0.25 & -2.99 \\
712.98 & 711.61 & 1.37 & 31 & -0.23 & -5.38 \\
733.95 & 732.36 & 1.59 & 32 & -0.15 & -5.52 \\
757.35 & 754.47 & 2.88 & 33 & 0.18 & -5.85 \\
778.92 & 776.99 & 1.93 & 34 & 0.10 & -5.02 \\
799.50 & 798.55 & 0.95 & 35 & 0.62 & -3.29 \\
821.15 & 820.45 & 0.7 & 36 & 0.78 & -3.22 \\
843.69 & 843.05 & 0.64 & 37 & 1.75 & -6.00 \\
- & 865.34 & - & 38 & 3.15 & -8.14 \\
885.74 & 886.67 & -0.93 & 39 & 3.68 & -7.76 \\
907.49 & 907.18 & 0.31 & 40 & 5.54 & -8.47 \\
- & 929.26 & - & 41 & 6.75 & -6.26 \\
951.75 & 952.70 & -0.95 & 42 & 8.44 & -4.84 \\
972.25 & 974.32 & -2.07 & 43 & 12.8 & -8.73 \\
994.39 & 994.46 & -0.07 & 44 & 16.5 & -11.76 \\
1016.47 & 1016.43 & 0.04 & 45 & 20.6 & -10.94 \\
1038.12 & 1038.84 & -0.72 & 46 & 24.8 & -11.12 \\
1058.03 & 1059.87 & -1.84 & 47 & 23.3 & -8.72 \\
- & 1081.23 & - & 48 & 25.5 & -8.25 \\
1103.29 & 1103.12 & 0.17 & 49 & 31.9 & -14.24 \\
1124.12 & 1124.68 & -0.56 & 50 & 27.8 & -13.66 \\
1146.35 & 1146.53 & -0.18 & 51 & 19.2 & -10.07 \\
- & 1167.32 & - & 52 & 11.2 & -12.32 \\
1189.96 & 1188.85 & 1.11 & 53 & -2.27 & -10.27 \\
\hline & & & & &
\end{tabular}

close to that suggested by spectroscopy. A detailed comparison of the observed $m=0$ periods of RX J2117.1+3412 with the theoretical periods of the best-fit model is given in Table 1 . The high quality of our period fit is quantitatively reflected by the average of the absolute period differences, $\overline{\delta \Pi_{i}}=$ $\left(\sum_{i=1}^{n}\left|\delta \Pi_{i}\right|\right) / n$, where $\delta \Pi_{i}=\Pi_{i}^{\mathrm{O}}-\Pi_{k}$, and by the root-meansquare residual, $\sigma_{\delta \Pi_{i}}=\sqrt{\left(\sum\left|\delta \Pi_{i}\right|^{2}\right) / n}$. We obtain $\overline{\delta \Pi_{i}}=1.08 \mathrm{~s}$ and $\sigma_{\delta \Pi_{i}}=1.34 \mathrm{~s}$. The quality of our fit for RX J2117.1+3412 is substantially better than those achieved by Kawaler \& Bradley (1994) and Córsico \& Althaus (2006) $\overline{\left(\delta \Pi_{i}\right.}=1.19 \mathrm{~s}$ and $\overline{\delta \Pi_{i}}=$ $1.79 \mathrm{~s}$, respectively) for PG $1159-035$.

Table 1 also shows the linear growth rates $\left(\eta_{k}\right)$ of the fitted pulsation modes (fifth column), computed with the linear, nonradial, nonadiabatic pulsation code described in Córsico et al. (2006). A positive value of $\eta_{k}$ implies pulsational instability. It is interesting to note that the domain of unstable-mode periods (757 $\lesssim \Pi_{k} \lesssim 1167$ s) of our best-fit model nearly coincides with the range of the observed periods for RX J2117.1+3412, although our calculations predict the observed modes with $k=30,31,32$ and 53 to be pulsationally stables, and conversely, modes such as $k=38,41,48$ and 52, which are unstable according to our predictions, are not observed at all in RX J2117.1+3412.

The last column in Table 1 shows the theoretical rate of period change of the fitted pulsation modes. Our calculations predict all pulsation periods to decrease with time $\left(\dot{\Pi}_{k}<0\right)$, an effect attributable to the rapid contraction experienced by the star in its evolution to the blue (see Fig. 1). Unfortunately, an unambiguous $\dot{\Pi}$ measurement for any mode of RX J2117.1+3412 is currently very difficult because of the large amplitude variations of the modes (VEA02). On the basis of considerations of the mass-loss rate and the helium-rich envelope mass in 

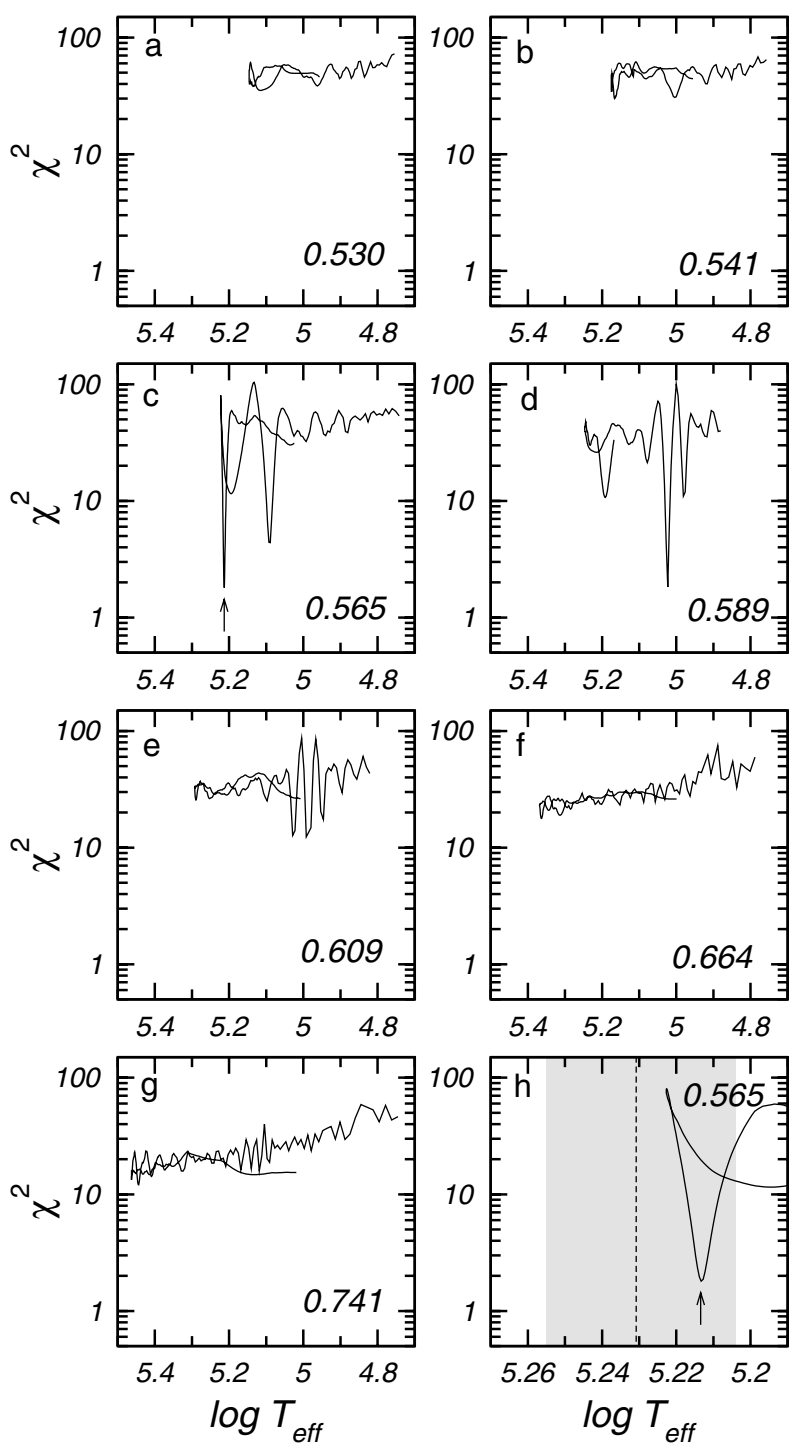

Fig. 4. The quality function of the period fit, $\chi^{2}$, in terms of $T_{\text {eff }}$ for the PG 1159 sequences with different stellar masses, indicated (in solar mass) at the right-bottom corner of each panel. Note the strong minima in panels c) and d), corresponding to $M_{*}=0.565 M_{\odot}$ and $M_{*}=0.589 M_{\odot}$, respectively. Panel h) is a zoom of the region with the strong minimum seen in panel c) (arrow). The vertical dashed line is the spectroscopic $T_{\text {eff }}$ of RX $2117.1+3412(170 \mathrm{kK})$ and the grey zone depicts its uncertainties $( \pm 10 \mathrm{kK})$.

RX J2117.1+3412, VEA02 give a rough estimation of the rate of period change of $|\dot{\Pi}| \sim 3-24 \times 10^{-11} \mathrm{~s} / \mathrm{s}$, much in agreement with our predictions.

\section{Discrepancy between spectroscopic and seismic mass}

The main features of our best-fit model are summarized in Table $2^{6}$, where we also include the parameters of RX J2117.1+3412 from other published studies. Note that the effective temperature of the best-fit model is compatible at $1 \sigma$ with the spectroscopically inferred value. According to our results, the temperature of $\mathrm{RX} \mathrm{J2117.1+3412} \mathrm{is} \mathrm{somewhat} \mathrm{lower}$

${ }^{6}$ Errors in $T_{\text {eff }}$ and $\log \left(L_{*} / L_{\odot}\right)$ are estimated from the width of the minimum in the function $\chi^{2}$ vs. $T_{\text {eff }}$ and $\log \left(L_{*} / L_{\odot}\right)$, respectively; the error in the stellar mass comes from the grid resolution in $M_{*}$. Errors in the other quantities are derived from these values.
Table 2. The main characteristics of the central star and the planetary nebula of RX J2117.1+3412. The second column corresponds to spectroscopic results, whereas the third and fourth columns present results from the pulsation study of VEA02 and from the asteroseismological model of this work, respectively.

\begin{tabular}{lccc}
\hline \hline Quantity & Spectroscopy & $\begin{array}{c}\text { Pulsations } \\
\text { (VEA02) }\end{array}$ & $\begin{array}{c}\text { Asteroseismology } \\
\text { (This work) }\end{array}$ \\
\hline$\overline{\Delta \Pi}[\mathrm{s}]$ & - & $21.618 \pm 0.008$ & 21.707 \\
\hline$T_{\mathrm{eff}}[\mathrm{kK}]$ & $170 \pm 10^{(\mathrm{a})}$ & - & $163.4_{-3.7}^{+2.5}$ \\
$M_{*}\left[M_{\odot}\right]$ & $0.72_{-0.12}^{+0.15(\mathrm{~b})}$ & $0.56_{-0.04}^{+0.02}$ & $0.565_{-0.023}^{+0.024}$ \\
$\log g\left[\mathrm{~cm} / \mathrm{s}^{2}\right]$ & $6.0_{-0.3}^{+0.3(\mathrm{a})}$ & - & $6.61_{-0.07}^{+0.11}$ \\
$\log \left(L_{*} / L_{\odot}\right)$ & $3.95 \pm 0.5^{(\mathrm{c})}$ & $4.05_{-0.32}^{+0.23}$ & $3.36 \pm 0.04$ \\
$\log \left(R_{*} / R_{\odot}\right)$ & - & $-0.91_{-0.15}^{+0.10}$ & $-1.23_{-0.025}^{+0.046}$ \\
$M_{\mathrm{env}}\left[M_{\odot}\right]$ & - & $0.0073-0.044$ & $0.02 \pm 0.006$ \\
$X_{\mathrm{He}}, X_{\mathrm{C}}, X_{\mathrm{O}}$ & $0.39,0.55,0.06$ & - & $0.39,0.32,0.22$ \\
$B C[\mathrm{mag}]$ & - & - & $-7.954_{-0.16}^{+0.01}$ \\
$M_{\mathrm{V}}[\mathrm{mag}]$ & - & - & $4.43_{-0.23}^{+0.12}$ \\
$A_{\mathrm{V}}[\mathrm{mag}]$ & - & 0.86 & $0.45_{-0.012}^{+0.036}$ \\
$d[\mathrm{pc}]$ & $1400_{-500}^{+700(\mathrm{c})}$ & $760_{-235}^{+230}$ & $452_{-23}^{+46}$ \\
$\pi[\mathrm{mas}]$ & $0.71_{-0.24}^{+0.40}$ & $1.32_{-0.30}^{+0.59}$ & $2.21_{-0.20}^{+0.12}$ \\
$t_{*}[\mathrm{yr}]$ & $2-5 \times 10^{4(\mathrm{~d})}$ & $>1.3 \times 10^{5}$ & $\gtrsim 2.5 \times 10^{4}$ \\
\hline$D_{\mathrm{PN}}[\mathrm{pc}]$ & $5.3_{-1.9}^{+2.7(\mathrm{~d})}$ & $2.9 \pm 0.9$ & $1.72_{-0.09}^{+0.18}$ \\
$t_{\mathrm{PN}}[\mathrm{yr}]$ & $\gtrsim 1.5 \times 10^{5(\mathrm{~d})}$ & $\sim 9.1 \times 10^{4 *}$ & $5.43_{-0.29}^{+0.57} \times 10^{4}$ \\
\hline
\end{tabular}

References: ${ }^{(a)}$ Werner et al. (1996); ${ }^{\text {(b) }}$ Miller Bertolami \& Althaus (2006); ${ }^{(c)}$ Motch et al. (1993); (d) Appleton et al. (1993). * This value is inferred from the linear size of the planetary nebula derived by VEA02 and a mean expansion velocity of the nebula of $31 \mathrm{~km} \mathrm{~s}^{-1}$.

than previous estimations, but it is still the hottest known PG 1159 pulsator. On the other hand, the best-fit model has a stellar mass of $M_{*}=0.565 M_{\odot}$, very close to the values found from the period spacing data (see Sect. 3). Thus, the linear pulsation theory strongly suggests that RXJ2117.1+3412 is an average-mass $P G 1159$ star with $M_{*} \sim 0.56-0.57 M_{\odot}$. This is clearly at odds with the spectroscopic-evolutionary predictions, that indicate a rather high mass of $0.70-0.72 M_{\odot}$ for this star (Werner \& Herwig 2006; Miller Bertolami \& Althaus 2006). A mass discrepancy is also encountered with other PG 1159 pulsators (PG 1159-035, PG 1707+427, PG 2131+066, PG0122+200). In those cases the seismic masses are always higher than the spectroscopic masses (see Córsico et al. 2006), however, the differences are a factor of three smaller (0.04-0.06 $\left.M_{\odot}\right)$ than for RX J2117.1+3412.

The mass discrepancy for RX J2117.1+3412 is reflected by the difference between the surface gravity of our pulsation model and the value predicted by spectroscopy. In fact, for our best-fit model we have $\log g=6.61$, large in excess as compared with the derived value of $\log g=6.0_{-0.2}^{+0.3}$ by using NLTE model atmospheres. Hence, the location of the star in the $\log T_{\text {eff }}-\log g$ plane shifts, according to our seismological predictions, downwards well beyond the limits of the observational error box (see Fig. 1).

It is obvious that the seismic results on $T_{\text {eff }}$ and $\log g$ are difficult to reconcile with results of model atmosphere analyses. This is demonstrated with Fig. 5, which shows a HST spectrum of RX J2117.1+3412 in the vicinity of the O V $1371 \AA$ line. This feature is very temperature sensitive and is therefore used as a strategic line to fix $T_{\text {eff }}$ in PG 1159 stars. It is not detectable in RX J2117.1+3412 which puts a tight lower limit to $T_{\mathrm{eff}}$. The lower panel of Fig. 5 shows a series of model profiles with 


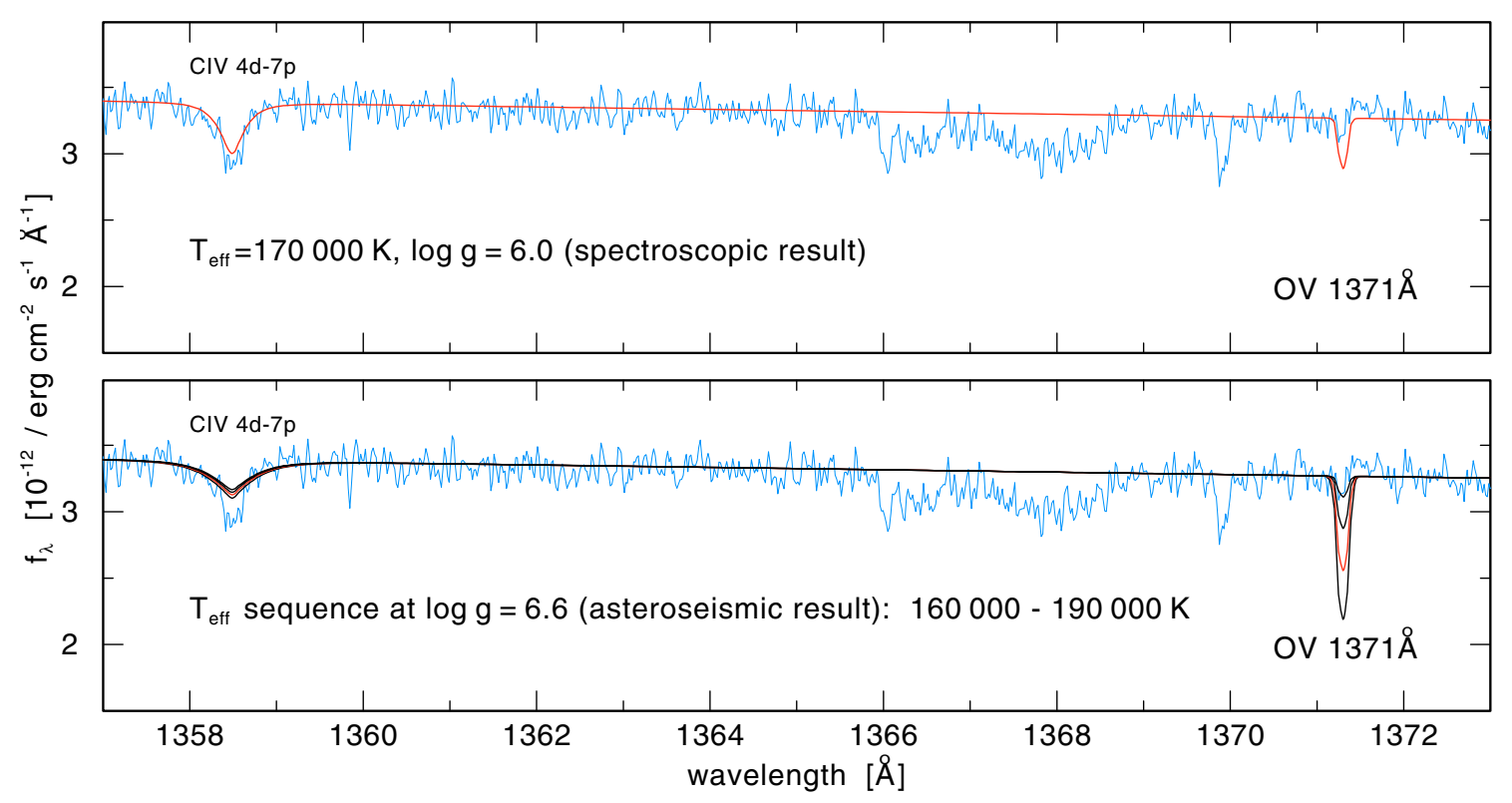

Fig. 5. Detail of a HST spectrum of RX J2117.1+3412 comprising a C IV line and the location of the $T_{\text {eff }}$ sensitive O V $1371 \AA$ line. The O V line is not detected, placing a lower limit to $T_{\text {eff }}$. Upper panel: comparison with a synthetic spectrum from a model with $T_{\text {eff }}=170 \mathrm{kK}$ and $\log g=6.0$, the best-fit parameters from optical and UV spectroscopy. At $T_{\text {eff }} \leq 170 \mathrm{kK}$ the model predicts a detectable O V line. Lower panel: comparison with four synthetic spectra with $T_{\text {eff }}=160,170,180,190 \mathrm{kK}$. The O v line gets weaker with increasing $T_{\text {eff }}$. The surface gravity is fixed at log $g=6.6$, the asteroseismic result. At this higher gravity, $T_{\text {eff }}$ must exceed $180 \mathrm{kK}$ in order to have non-detectable $\mathrm{OV}$ line, significantly above the asteroseismic result of $T_{\mathrm{eff}}=163 \mathrm{kK}$.

increasing $T_{\text {eff }}$, whereas the gravity is kept fixed at the asteroseismic value $\log g=6.6$. The model atmospheres were calculated with the Tübingen Model Atmosphere Package (see, for details, Werner et al. 2003). The model with $T_{\text {eff }}=160 \mathrm{kK}$ (a value close to the asteroseismic result of $163 \mathrm{kK}$ ) produces a strong $\mathrm{O} \mathrm{V}$ line, clearly contradicting the observation. A model temperature of $190 \mathrm{kK}$ is necessary to make the line undetectably weak. In the upper panel we show the model with the spectroscopically determined best-fit parameters $\left(T_{\text {eff }}=170 \mathrm{kK}, \log g=6.0\right)$. In the analysis of Werner et al. (1996) we regarded this $T_{\text {eff }}$ as a lower limit but excluded temperatures higher than $180 \mathrm{kK}$ because all He II and C IV lines in the optical and UV become too shallow. This discrepancy between spectroscopic and asteroseismic results calls for a comprehensive re-analysis taking into account all available spectroscopic material collected since the Werner et al. (1996) analysis.

VEA02 infer the stellar luminosity and radius of RX J $2117.1+3412$ on the basis of their mass estimation and the effective temperature and gravity measured by spectroscopy. Thus, not surprisingly, their results point to a larger luminosity and radius $\left(\log L_{*} / L_{\odot}=4.05_{-0.32}^{+0.23}\right.$ and $\left.\log \left(R_{*} / R_{\odot}\right)=-0.91_{-0.15}^{+0.10}\right)$ as compared with our best-fit model $\left(\log L_{*} / L_{\odot}=3.36\right.$ and $\left.\log \left(R_{*} / R_{\odot}\right)=-1.23\right)$.

\section{Helium envelope thickness}

Another important parameter is the thickness of the outer envelope $\left(M_{\text {env }}\right)$ of RX J2117.1+3412. We define $M_{\text {env }}$ as the mass above the chemical discontinuity between the He-rich envelope and the $\mathrm{C} / \mathrm{O}$ core (see Fig. 6). Our best-fit model has $M_{\text {env }}=0.02 M_{\odot}^{7}$. VEA02 claim that $M_{\text {env }}$ is $0.0073-0.044 M_{\odot}$, which is in line with our results. It is important to note

\footnotetext{
7 We define the "effective" location of the $\mathrm{He} / \mathrm{C} / \mathrm{O}$ chemical interface as the position of the layer characterized by an helium abundance of $X_{\mathrm{He}} \sim 0.19$. In our best-fit model this corresponds at $M_{r} \sim 0.544 M_{\odot}$
}

that VEA02 derive these values of $M_{\text {env }}$ on the basis of the results published by Kawaler \& Bradley (1994) for PG 1159-035, extrapolated to the range of parameters of RX J2117.1+3412. Specifically, they use a relation connecting the fractional mass of the helium-rich envelope with the effective temperature and the trapping cycle for PG 1159-035 and RX J2117.1+3412. The underlying assumption is that the mode-trapping features of PG 1159 stars are inflicted mainly by the outer chemical interface of $\mathrm{He} / \mathrm{C} / \mathrm{O}$, and thus, the minima seen in the periodspacing distribution are associated with modes trapped in the envelopes. However, realistic evolutionary calculations predict mode-trapping properties of PG 1159 models to be primarily fixed by the stepped structure of the $\mathrm{C} / \mathrm{O}$ chemical profile at the core left by prior extra mixing episodes (Fig. 6), the outer $\mathrm{He} / \mathrm{C} / \mathrm{O}$ interface playing a secondary role (see Córsico $\&$ Althaus 2005, 2006). For instance, the observed periods at $799.50 \mathrm{~s}(k=35)$ and $1058.03 \mathrm{~s}(k=47)$, which correspond to modes trapped in the envelope of RX J2117.1+3412 according VEA02, are modes "confined" to the core region in our best-fit model and thus not related to the thickness of the helium-rich outer envelope. Thus, the procedure from which VEA02 derived the thickness of the helium-rich outer envelope of RX J2117.1+3412 should be taken with some caution.

The employment of full PG 1159 evolutionary models allows us to place constraints on the amount of stellar mass eroded by winds. We can do this by adopting typical mass loss rates for the different stages after the VLTP. For the short superwind phase, that lasts $\sim 800 \mathrm{yr}$ and takes place at $\log T_{\text {eff }}<3.8$, we adopt $10^{-5} M_{\odot} / \mathrm{yr}$ as observed at Sakurai's Object (Tyne et al. 2002). For the evolution before the star reaches $T_{\text {eff }} \sim 100000 \mathrm{~K}$ (this stage takes $\sim 2000 \mathrm{yr}$ ) we adopt typical [WR] planetary nebula nuclei mass loss rates of $10^{-6} M_{\odot} / \mathrm{yr}$ (Koesterke 2001). Finally, for the PG 1159 stage we can adopt the observed value

(see the vertical line in Fig. 6), where the contribution of the $\mathrm{He} / \mathrm{C} / \mathrm{O}$ chemical interface to the Brunt-Väisälä frequency is largest. 


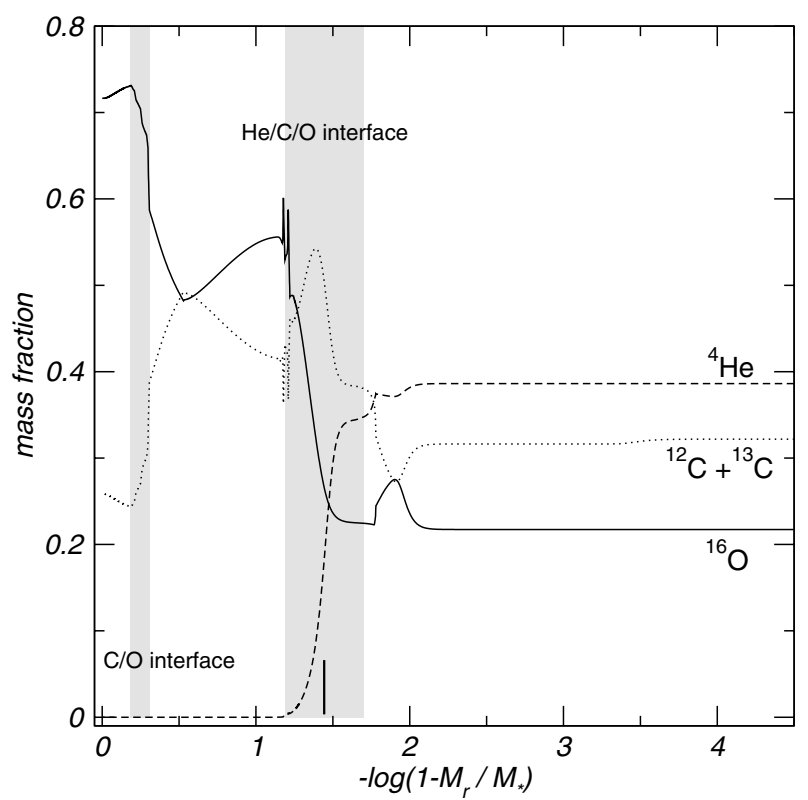

Fig. 6. The internal chemical profile of our best-fit model in terms of the fractional mass coordinate. The thickness of the outer envelope is $M_{\text {env }}=0.02 M_{\odot}$. The location of the $\mathrm{C} / \mathrm{O}$ and $\mathrm{He} / \mathrm{C} / \mathrm{O}$ chemical transition regions is emphasized with grey regions. The vertical line at $-\log \left(1-M_{r} / M_{*}\right) \sim 1.4$ marks the location of the layer where $X_{\mathrm{He}} \sim 0.19$.

of $4 \times 10^{-8} M_{\odot} / \mathrm{yr}$ (during the $12000 \mathrm{yr}$ until the sequence reaches the best fit model). Under these assumptions we estimate the possible mass lost by the star after the VLTP to be $10^{-2} M_{\odot}$, which is half of the mass of the He-rich envelope. Thus the remaining envelope will be still thick enough for it not to affect the results of the present work. Even more, as the evolution of the star would be accelerated by these mass loss episodes, the previous estimation represents an upper limit under the adopted mass loss rates.

\section{The seismic distance to $\mathrm{RX} \mathbf{J} 2117.1+3412$ and the implications for its planetary nebula}

In closing, we infer the seismic distance of RX J2117.1+3412 from the Earth. First, we consider the flux predicted by a NLTE model atmosphere with $T_{\text {eff }}=160 \mathrm{kK}$ and $\log g=6.6$ integrated through the spectral response of the $\mathrm{V}$ filter. We obtain a bolometric correction $B C=-7.954$ and an absolute magnitude $M_{\mathrm{v}}=4.43$. Because the proximity of RX J2117.1+3412 to the galactic plane, we must account for the interstellar absorption, $A_{\mathrm{V}}$. In line with VEA02, we follow the interstellar extinction model developed by Chen et al. (1998). We compute the seismic distance $d$ according to the well-known relation:

$\log d=\frac{1}{5}\left[m_{\mathrm{v}}-M_{\mathrm{v}}+5-A_{\mathrm{V}}(d)\right]$,

where the apparent magnitude is $m_{\mathrm{v}}=13.16 \pm 0.01$ (Motch et al. 1993). The interstellar absorption $A_{\mathrm{V}}(d)$ varies non linearly with the distance and also depends on the Galactic latitude $(b)$ and longitude $(\ell)$. For the equatorial coordinates of RX J2117.1+3412 (Epoch B2000.00, $\alpha=21^{\mathrm{h}} 17^{\mathrm{m}} 7.60, \delta=$ $+34^{\circ} 12^{\prime} 22^{\prime \prime}$. 0 ) the corresponding Galactic coordinates are $b=$ $-10^{\circ} 24^{\prime} 32^{\prime \prime}$.04 and $\ell=80^{\circ} 21^{\prime} 10^{\prime}$. 8 . We solve Eq. (1) iteratively and obtain a distance $d=452 \mathrm{pc}$ and an interstellar extinction $A_{\mathrm{V}}=0.45$, substantially lower than the estimations of VEA02 ( $d=760_{-235}^{+230} \mathrm{pc}$ and $\left.A_{\mathrm{V}}=0.86\right)$. This is due to the fact that the luminosity of our model $\left(2.3 \times 10^{3} L_{\odot}\right)$ is considerably lower than the luminosity adopted by those authors $\left(1.1 \times 10^{4} L_{\odot}\right)$.

Our calculations predict a parallax of $\sim 2.21$ mas and a linear diameter of the planetary nebula of $\sim 1.72 \mathrm{pc}\left(5.3 \times 10^{13} \mathrm{~km}\right)$. If we assume a mean expansion velocity for a typical planetary nebula with a WR-type central star of approximately $31 \mathrm{~km} \mathrm{~s}^{-1}$ (Gorny \& Stasinska 1995), then the expansion age for the nebula would be $t_{\mathrm{PN}} \approx 5.43 \times 10^{4} \mathrm{yr}$. On the other hand, our computations predict an evolutionary age for the best-fit VLTP model of about $t_{*} \approx 2.5 \times 10^{4} \mathrm{yr}$ and possible larger if the VLTP would have occurred later on the hot white dwarf cooling branch than in our sequence ${ }^{8}$. This age is well consistent (to a factor less than two) with the expansion age of the nebula. Thus, our results seem to solve the apparent inconsistency between the evolutionary timescale of RXJ2117.1+3412 and the size of the nebula (see Appleton et al. 1993, for details). The agreement between $t_{*}$ and $t_{\mathrm{PN}}$ according to the present calculations reinforces the validity of our asteroseismological model and may suggest that RX J2117.1+3412 have undergone a VLTP episode, a fact that would be at variance with the lack of ${ }^{14} \mathrm{~N}$ in its atmosphere.

\section{Summary and conclusions}

In this paper we carried out an asteroseismological analysis of the hot pulsating PG 1159 star RX J2117.1+3412, a $g$-mode pulsator with properties intermediate between young planetary nebula central stars and PG 1159 stars without planetary nebula. Our analysis is based on the full PG 1159 evolutionary models of Miller Bertolami \& Althaus (2006). These models represent a solid basis to analyze the evolutionary and pulsational status of hot PG 1159 stars like RX J2117.1+3412, a transition object for which stellar models extracted from artificial evolutionary procedures are not appropriate. We first made good use of the fact that the period spacing of variable PG 1159 stars is mainly a function of the stellar mass, and derived a value of $M_{*} \sim 0.568 M_{\odot}$ by comparing $\Delta \Pi^{\mathrm{O}}$ with the asymptotic period spacing of our models. We also compared $\Delta \Pi^{\mathrm{O}}$ with the computed period spacing averaged over the period range observed in RX J2117.1+3412, and derived a value of $M_{*} \sim 0.560 M_{\odot}$. Note that in both derivations of the stellar mass we made use of the spectroscopic constraint that the effective temperature of the star should be of $\sim 170 \mathrm{kK}$.

Next, we adopted a less conservative approach in which the individual pulsation periods alone naturally lead to an "asteroseismological" PG 1159 model that is assumed to be representative of RX J2117.1+3412. The period fit was made on a grid of PG 1159 models with a quite fine resolution in effective temperature $\left(\Delta T_{\text {eff }} \sim 10-30 \mathrm{~K}\right)$ although admittedly coarse in stellar mass $\left(\Delta M_{*} \sim 0.012-0.077 M_{\odot}\right)$. The match between the computed dipole pulsation periods of the best-fit model and the observed periods in RX J2117.1+3412 is excellent, with an average of the absolute period differences of $1.08 \mathrm{~s}$ and a rootmean-square residual of $1.34 \mathrm{~s}$. It is worth noting also that the domain of unstable-mode periods of the best-fit model nearly matches the range of the observed periods in RX J2117.1+3412, although there is no obvious correlation between the magnitude of the linear growth rates and the observed mode amplitudes.

${ }^{8}$ We mention that our $0.565 M_{\odot}$ sequence predicts a time interval between the occurrence of the VLTP and the present state of RX J2117.1+3412 of $\approx 1.5 \times 10^{4} \mathrm{yr}$. 
Interestingly enough, the mass of the best-fit model $\left(M_{*}=\right.$ $\left.0.565 M_{\odot}\right)$ agrees with the mass derived from the observed period spacing, but it is $\sim 25 \%$ lower than the value $M_{*}=0.72 M_{\odot}$ derived from spectroscopy coupled with our evolutionary tracks.

Other characteristics of the best-fit model are summarized in Table 2. In particular, the effective temperature is compatible at $1 \sigma$ with the spectroscopic value. At variance with this, the surface gravity of the best-fit model is substantially higher than that given by spectroscopy. We also infer the "seismic distance" of RX J2117.1+3412 and obtain a distance $d \sim 452 \mathrm{pc}$, which places the star markedly closer to the Earth than thought hitherto $(d \sim 760-1400$ pc, Vauclair et al. 2002 and Motch et al. 1993). The derived distance implies a linear size of the nebula of $1.72 \mathrm{pc}$ which implies an expansion age of $\approx 5.43 \times 10^{4} \mathrm{yr}$. This age is substantially lower than assumed hitherto (Appleton et al. 1993) and in better agreement with the times predicted by evolutionary models. This result reinforces the correctness of our asteroseismological model for RX J2117.1+3412.

Finally, our computations predict a temporal period drift for RX J2117.1+3412 between $-3 \times 10^{-11} \mathrm{~s} / \mathrm{s}$ and $-1.4 \times 10^{-10} \mathrm{~s} / \mathrm{s}$. The negative values of $\dot{\Pi}$ reflect the fact that our best-fit model is still rapidly contracting on its evolutionary road towards higher effective temperatures in the HR diagram. Unfortunately, the amplitude variability of the observed modes in RX J2117.1+3412 precludes any measurement of $\dot{\Pi}$ for the moment, thus hindering any test of our prediction.

The results of the asteroseismological analysis carried out in this work strongly suggest that the mass of RX J2117.1+3412 is considerably lower than suggested by spectroscopy coupled to evolutionary tracks (Werner \& Herwig 2006; Miller Bertolami $\&$ Althaus 2006). This serious disagreement is also seen in other pulsating PG 1159 stars like PG 1159-035, PG 1707+427, PG 2131+066 and PG0122+200, although in those cases the seismic masses are always higher than the spectroscopic masses (Córsico et al. 2006). The discrepancy could be attributed to a number of factors. On the observational side, possible errors in the spectroscopic determinations of $g$ and $T_{\text {eff }}$; in particular, as this study suggests in the case of RX J2117.1+3412, in the determination of $g$. In this respect, detailed asteroseismological fits to other pulsating PG 1159 stars would be valuable. On the theoretical front, different PG 1159 evolutionary tracks could result from different dredge-up and/or mass loss history in the AGB progenitor evolution (Werner \& Herwig 2006). But in preliminary simulations we have found that neither third dredge up efficiency nor TP-AGB lifetimes play a determining role in the location of PG 1159 tracks. It remains to be seen if other assumptions in the microphysics such as radiative and conductive opacities and/or equation of state may be playing a role in the location of post-AGB tracks.

Acknowledgements. We warmly acknowledge our referee (S. O. Kepler) for his comments and suggestions which strongly improved the original version of the paper. We thank Rubén Martínez and Héctor Viturro for valuable technical support. M.M.M.B. thanks an EARA-EST from the European Association for Research in Astronomy. This research was partially supported by the PIP 6521 grant from CONICET.

\section{References}

Alexander, D. R., \& Ferguson, J. W. 1994, ApJ, 437, 879

Allen, C. W., ed. 1973, Astrophysical Quantities, 3d edn. (London: Athlone)

Althaus, L. G., Serenelli, A. M., Panei, J. A., et al. 2005, A\&A, 435, 631

Appleton, P. N., Kawaler, S. D., \& Eitter, J. J. 1993, AJ, 106, 1973

Blöcker, T. 2001, Ap\&SS, 275, 1

Chen, B., Vergely, J. L., Valette, B., \& Carraro, G. 1998, A\&A, 336, 137

Córsico, A. H., \& Althaus, L. G. 2005, A\&A, 439, L31

Córsico, A. H., \& Althaus, L. G. 2006, A\&A, 454, 863

Córsico, A. H., Althaus, L. G., \& Miller Bertolami, M. M. 2006, A\&A, 458, 259 Gautschy, A. 1997, A\&A, 320, 811

Gorny, S. K., \& Stasinska, G. 1995, A\&A, 303, 893

Hajduk, M., Zijlstra, A., Herwig, F., et al. 2005, Science, 308, 231

Herwig, F. 2001, ApJ, 554, L71

Iglesias, C. A., \& Rogers, F. J. 1996, ApJ, 464, 943

Kawaler, S. D., \& Bradley, P. A. 1994, ApJ, 427, 415

Koesterke, L. 2001, Ap\&SS, 275, 41

Koesterke, L., \& Werner, K. 1998, ApJ, 500, L55

Koesterke, L., Dreizler, S., \& Rauch, T. 1998, A\&A, 330, 1041

Lawlor, T. M., \& MacDonald, J. 2003, ApJ, 583, 913

Miller Bertolami, M. M., \& Althaus, L. G. 2006, A\&A, 454, 845

Miller Bertolami, M. M., Althaus, L. G., Serenelli, A. M., \& Panei, J. A. 2006, A\&A, 449, 313

Motch, C., Werner, K., \& Pakull, M. W. 1993, A\&A, 268, 561

Nather, R. E., Winget, D. E., Clemens, J. C., Hansen, C. J., \& Hine, B. P. 1990, ApJ, 361, 309

Rauch, T., \& Werner, K. 1997, in The Third Conference on Faint Blue Stars, ed. A. G. D. Philip, J. Liebert, \& R. A. Saffer (Schenectady, NY: L. Davis Press), 217

Schönberner, D. 1979, A\&A, 79, 108

Tassoul, M., Fontaine, G., \& Winget, D. E. 1990, ApJS, 72, 335

Tyne, V. H., Evans, A., Geballe, T. R., et al. 2002, MNRAS, 334, 875

Vauclair, G., Belmonte, J. A., Pfeiffer, B., et al. 1993, A\&A, 267, L35

Vauclair, G., Moskalik, P., Pfeiffer, B., et al. 2002, A\&A, 381, 122 (VEA02)

Watson, T. K. 1992, IAU Circ., 5603

Werner, K., \& Herwig, F. 2006, PASP, 118, 183

Werner, K., Dreizler, S., Heber, U., et al. 1996, A\&A, 307, 860

Werner, K., Deetjen, J. L., Dreizler, S., et al. 2003, in Stellar Atmosphere Modeling, ed. I. Hubeny, D. Mihalas, \& K. Werner, ASP Conf. Ser., 288, 31

Wood, P. R., \& Faulkner, D. J. 1986, ApJ, 307, 659 